Evelin Edith Aquino Rojas Lizzeth Pamela Gamboa Ortuño

Javier Alexander Gueresa Mikael Agreda Herbas

Correspondencia a:

Centro de investigación medicina UNITEPC. CIMU

Cochabamba - Bolivia

eaquino_investigacion@ unitepc-mail.com

lizzeth_gamboa@unitepc-mail.com

jalexander_g@unitepc-mail.com

mikael_agreda@unitepc-mail.com

Procedencia y arbitraje: No comisionado, sometido a arbitraje externo

Recibido para publicación: 24 de mayo de 2018 Aceptado para publicación: 17 de septiembre de 2018

\section{FUENTES PREDOMINANTES DE LA COLONIZACIÓN DE AEDES AEGYPTI QUE FACILITA EL PROCESO DE DISPER- SIÓN EN EL MUNICIPIO DE COLCAPIRHUA}

\section{PREDOMINANT SOURCES OF THE COLONIZATION OF AE- DES AEGYPTI THAT FACILITATES THE DISPERSION PRO- CESS IN THE MUNICIPALITY OF COLCAPIRHUA}

FONTES PREDOMINANTES DA COLONIZAÇÃO DE AEDES AEGYPTI QUE FACILITA O PROCESSO DE DISPERSÃO NO MUNICÍPIO DE COLCAPIRHUA

\section{RESUMEN}

Los hallazgos del presente estudio desarrollado en el municipio de Colcapirhua confirman la dispersión del A. aegypti mediante la geo referenciación de los focos identificados del vector, con algunos sectores con mayor prevalencia. De la misma forma, se evidencia que la dispersión del vector en el área de estudio alcanzo una altura mínima de $2538 \mathrm{~m} / \mathrm{s} / \mathrm{n} / \mathrm{m}$. y una altura máxima de $2623 \mathrm{~m} / \mathrm{s} / \mathrm{n} / \mathrm{m}$.

De 100 casas inspeccionados en el municipio de Colcapirhua; $52 \%$ son positivas relacionado a la presencia de depósitos desechables que sirven de criadero al vector. En todos los depósitos estudiados se detectó focos de A. aegypti. con mayor predominancia en llantas, turriles y depósitos desechables.

Las personas que viven en Colcapirhua tienen 3 veces más riesgo de albergar al vector en sus casas (RR 3.1 ; IC: .1,8 - 5,3; $\mathrm{X}^{2}$ 13,5; p: 0,00009)

Palabras claves: Aedes aegypti, Fuentes, Riesgo, Colonización, Colcapirhua.

\section{SUMMARY}

The findings of the present study developed in the municipality of Colcapirhua confirm the dispersion of A. aegypti by georeferencing the identified foci of the vector, with some sectors with higher prevalence. In the same way, it is evident that the dispersion of the vector in the study area reached a minimum height of $2538 \mathrm{~m} / \mathrm{s} / \mathrm{n} / \mathrm{m}$. and a maximum height of $2623 \mathrm{~m} / \mathrm{s} / \mathrm{n} /$ m. Of 100 Houses inspected in the municipality of Colcapirhua; $52 \%$ are positive related to the presence of disposable deposits that serve as a hatchery to the vector. Foci of $A$. aegypti were detected in all the deposits studied. with greater predominance in tires, turriles and disposable deposits. People living in Colcapirhua have 3 times more risk of harboring the vector in their homes (RR 3.1; Cl 1.8 - 5.3, X2 13.5, p: 0.00009).

Key words: Aedes aegypti, Sources, Risk, Colonization, Colcapirhua. 


\section{RESUMO}

Os achados do presente estudo, desenvolvido no município de Colcapirhua, confirmam a dispersão de A. aegypti por georreferenciar os focos identificados do vetor, com alguns setores com maior prevalência. Da mesma forma, é evidente que a dispersão do vetor na área de estudo atingiu uma altura mínima de $2538 \mathrm{~m} / \mathrm{s} / \mathrm{n} / \mathrm{m}$. e uma altura máxima de $2623 \mathrm{~m} / \mathrm{s} / \mathrm{n} / \mathrm{m}$.

Das 100 casas inspecionadas no município de Colcapirhua; $52 \%$ são positivos relacionados à presença de depósitos descartáveis que servem como incubatório para o vetor. Focos de A. aegypti foram detectados em todos os depósitos estudados. Com maior predominância em pneus, tambores e depósitos descartáveis.

As pessoas que vivem em Colcapirhua têm 3 vezes mais risco de abrigar o vetor em suas casas (RR 3.1, Cl: .1.8 - 5.3, X2 13.5, p: 0.00009)

Palavras chave: Aedes aegypti, Fontes, Risco, Colonização, Colcapirhua.

\section{INTRODUCCIÓN:}

Aedes es un género de mosquitos culícidos transmisores de varias enfermedades. ${ }^{1,2,3}$ Aedes aegypti (diptera:culicidae) o mosquito de Egipto; es un insecto díptero de la familia de los culícidos, conocido por el vulgo como "zancudo" o "patas blancas" $6,7,84,5$.

El mosquito A. aegypti, tiene su origen en el cinturón tropical de África donde generalmente se encuentran las especies del subgénero Stegomyia. 9,10,11,12,13

Esta especie se caracteriza por su preferencia por climas cálidos (de 15 a $40{ }^{\circ} \mathrm{C}$ ) y con niveles de precipitación pluvial moderados y altos, donde se generan condiciones ambientales favorables para su reproducción. 9,10,14,15,16,17, El mosquito está bien adaptado a ambientes domésticos y peri domiciliados, debido principalmente al hábito hematofágico de las hembras.

En el continente americano el mosquito A. aegypti es transmisor de enfermedades como el dengue chikungunya y zika; causa severos daños en la salud pública en las Américas y el mundo. ${ }^{18,20,21}$

Las enfermedades del dengue, zika, chikungunya y otros Flavivirus transmitidos por A. Aegypti, en la actualidad se convirtió en un problema creciente para la Salud Pública mundial, debido a múltiples factores: como el cambio y la variabilidad climática, aumento de la población mundial en áreas urbanas con crecimiento rápido y desorganizado, ${ }^{18,22,23}$ la insuficiente provisión de agua potable que obliga a su almacenamiento en recipientes caseros habitualmente descubiertos, ${ }^{24,25,26,27}$ la inadecuada recolección de residuos y la gran producción de recipientes descartables que sirven como criaderos de mosquitos al igual que los neumáticos desechados. ${ }^{28,29,30,31,32}$ Y sumándose a estos factores el aumento de viajes y migraciones, fallas en el control de los vectores y la falta de una vacuna eficaz para prevenir las enfermedades. ${ }^{33,35,37}$

Las características que distingue A. aegypti de otras especies de mosquitos es su habilidad de completar su desarrollo pre adulto $38,39,40$. en una gran variedad de recipientes naturales y artificiales, ${ }^{41,42,43}$ esto unido al aumento acelerado en el número de criaderos larvales generados por la actividad humana como consecuencia de pa- 
trones culturales, tradicionales y de falta de conocimiento, garantiza una permanente disponibilidad de criaderos potenciales para esta especie.

La expansión a nuevas aéreas por A. aegyti, donde antes era desconocido, muestra la plasticidad de la especie en la capacidad de dispersión aérea de Cochabamba. La infestación de viviendas por A. aegypti en el municipio de Colcapirhua de la ciudad de Cochabamba, no está del todo claro y genera una pregunta científica clave con vastas implicancias para la prevención y el control vectorial todavía no resuelta, es su capacidad de adaptación, colonización y dispersión del mosquito. Otro problema que agrava esta situación es la falta de recurso humano en las acciones de prevención y control vectorial para cubrir extensas áreas pobladas donde está presente el vector.

\section{METODOLOGÍA}

\section{TIPO DE ESTUDIO}

Es un estudio: no probabilístico aleatoria estratificado

El tipo de estudio epidemiológico: analítico no experimental, de estudio transversal analítico con una variable cuali - cuantitativa de escala nominal

\section{EVALUACIÓN ENTOMOLÓGICA}

La encuesta larvaria durante los ciclos de inspección que inicio en febrero de 2016 y concluyo a inicios de 2017, se basó en la búsqueda de los criaderos potenciales (depósitos con agua) para el $A$. aegypti. La búsqueda se realizó dentro y fuera de las casas. Los depósitos se clasificaron en 10 tipos: 1) tanques elevados; 2) tanques bajos (albercas); 3) barriles; 4) recipientes de barro; 5) latas; 6) botellas; 7) llantas; 8) árboles y plantas; 9) otros pequeños (floreros, bebederos de animales); 10) miscelánea (canales de desagüe en los techos, chatarras. Etc.).

Las evaluaciones entomológicas se las realizo en las viviendas seleccionadas utilizando los siguientes instrumentos: cucharon, pipetas, planillas de recolección de datos, tubos falcón, pinzas, linternas, GPS, etc. las muestras larvarias fueron acondicionadas en tubos falcón para su posterior confirmación en laboratorio mediante microscopios estereoscópicos.

Las planillas de evaluación entomológica fueron las mismas utilizadas por el Programa Nacional de Dengue.

Los indicadores a ser utilizados para este estudio fueron los siguientes:

\section{ÍNDICE DE INFESTACIÓN AEDICA DE LARVAS}

Porcentaje de casas infestadas con larvas de A. aegypti, en una determinada área o localidad. Mide la dispersión del vector en la localidad ${ }^{44,45,46.47}$

$$
\begin{aligned}
& \mathrm{N}^{\circ} \text { Viviendas positivas } \\
& \mathrm{II}=\frac{\mathrm{N}=\mathrm{N}}{\mathrm{N}{ }^{0} \text { viviendas inspeccionadas }}
\end{aligned}
$$

\section{ÍNDICE DE INFESTACIÓN AEDICA DE RECIPIENTES}

Porcentaje de depósitos con agua, infestado por larvas y pupas de A. aegypti en una 
localidad o área. ${ }^{48,49,50,51}$

$$
\mathrm{IIR}=\frac{\mathrm{N}^{0} \text { recipientes positivos }}{\mathrm{N}^{0} \text { recipientes inspeccionados }} \times 100
$$

\section{ÍNDICE DE INFESTACIÓN DE BRETEAU}

Porcentaje de recipientes positivos en las casas inspeccionadas de la localidad; mide la productividad de larvas por vivienda inspeccionada. ${ }^{52,53,54}$.

$$
\mathrm{IIB}=\frac{\mathrm{N}^{0} \text { recipientes positivos }}{\mathrm{N}^{0} \text { viviendas inspeccionadas }}
$$

\section{INDICADORES DE RIESGO ENTOMOLÓGICOS}

Para determinar el riesgo epidemiológico de las casas infestadas por A. aegypti, se tuvieron en cuenta los indicadores entomológicos: índices de infestación aédica, de recipiente y breteau, según escala en tabla a seguir. ${ }^{55,56,5,58 .}$

\section{CUADRO № 1}

\section{CALCIFICACIÓN DEL NIVEL DE RIESGO SEGÚN LOS INDICADORES ENTOMO- LÓGICOS}

\begin{tabular}{|l|l|l|l|}
\hline $\begin{array}{l}\text { NIVEL DE } \\
\text { CONTROL OPERA- } \\
\text { TIVO }\end{array}$ & $\begin{array}{l}\text { ÍNDICE DE } \\
\text { CASAS } \\
\text { POSITIVAS }\end{array}$ & $\begin{array}{l}\text { DE } \\
\text { RECIPIENTES PO- } \\
\text { SITIVOS }\end{array}$ & $\begin{array}{l}\text { IEDICE } \\
\text { BRETEAU }\end{array}$ \\
\hline OPTIMO & $<1$ & $<0.5$ & $1-4.9$ \\
\hline BUENO & $1-4.9$ & $0.5-1.9$ & $5-9.9$ \\
\hline $\begin{array}{l}\text { ALARMA } \\
\text { EMERGENCIA }\end{array}$ & $5-9.9$ & $2-4.9$ & $\begin{array}{l}10-14.9 \\
10 \text { a mas mas }\end{array}$ \\
\hline
\end{tabular}

FUENTE: OPS/OMS ${ }^{51}$

\section{ANÁLISIS ESPACIAL (GIS).}

La geo referenciación de los locales de presencia y ausencia de A. aegypti será realizo con ayuda de un GPS MAP 76CSX (Garmin $\left.{ }^{\circledR}\right)$. De la misma forma para el estudio fueron considerados datos entomológicos.

\section{SELECCIÓN DE LA MUESTRA}

Una vez calculado el tamaño de la muestra de 100 viviendas se procedió a la selección de los hogares mediante un tipo de muestreo probabilístico de diseño polimetálico; en el cual se realizó 


\section{ESQUEMA No 1}

\section{ESQUEMA DEL DISEÑO Y RECOLECCIÓN DE LA MUESTRA}

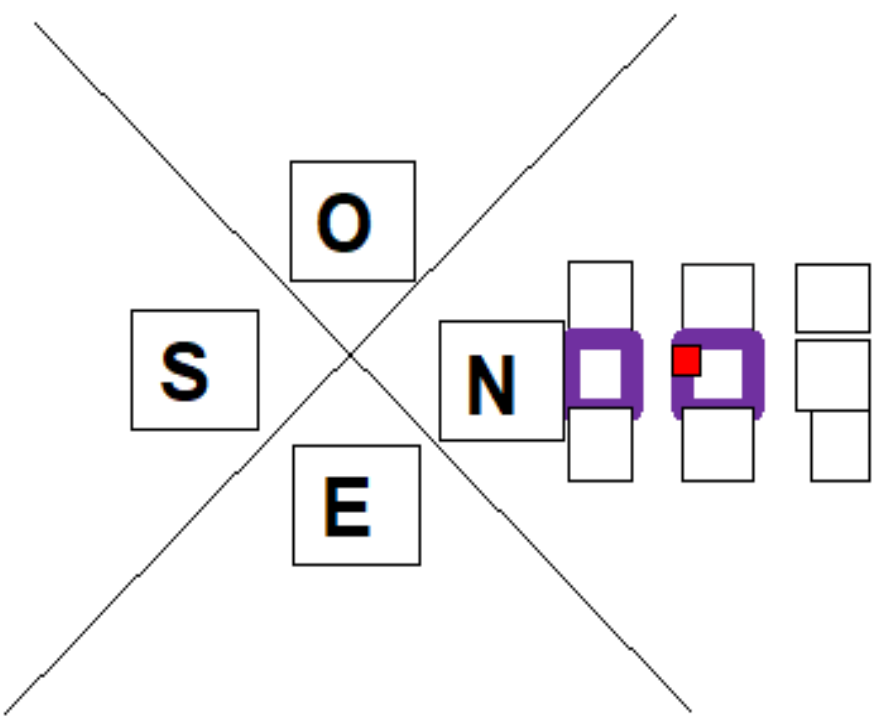

TOTAL, CASAS A 100

- Unidad de muestra: casa

- Tipo de muestra: probabilístico

- Diseño de muestra: polietapico

1)

M estratificado:

Municipio de Colcapirhua 6 sectores

2) M. por conglomerados $=66$

En cada sector del municipio son aproximadamente de $\mathbf{4}$ a $\mathbf{1 1}$ conglomerados

1 conglomerado es = a 1 manzano

3) M. estratificada dentro de los conglomerados

Por manzano tendremos 4 casas

\section{PLAN DE ANÁLISIS}

Esta información obtenida de los instrumentos aplicados se ingresó a Epi Info (2007), de acuerdo a las variables previamente descritas como sexo, edad, lugar de procedencia, lugar donde vive, etc.

De las variables de interés se calculó intervalo de confianza (IC) del 95\% asumiendo la normalidad y utilizando el método exacto para proporciones pequeñas

Para datos independientes y para posibles asociaciones entre variables cualitativas se utilizó el test de $\mathrm{X}^{2 ;}$ se considera estadísticamente significativas los valores de $\mathrm{P}<$ 0,05 .

Para determinar las características del brote de infestación del A. aegypti se realizó el riesgo relativo (RR), admitiendo significación estadística en el análisis bivariado.

Las hipótesis planteadas en los análisis estadísticos son los siguientes:

A. $\mathrm{X}^{2:} \mathrm{H}_{\mathrm{o}}=$ No influye la localización de la vivienda en el municipio como factor de riego.

$H_{A}=$ Sl influye la localización de la vivienda en el municipio como factor de riego. 
$\mathrm{X}^{2:} \mathrm{H}_{\mathrm{o}}$ No influyen los depósitos pequeños desechables a manera de recipientes, como factor de riesgo para hospedar al mosquito A.aegypti

$\mathrm{H}_{\mathrm{A}}=\mathrm{Si}$ influyen los depósitos pequeños desechables a manera de recipientes, como factor de riesgo para hospedar al mosquito A.aegypti

B. $\mathrm{X}^{2:} \mathrm{H}_{\mathrm{o}}$ No influyen las llantas a manera de recipientes, como factor de riesgo para hospedar al vector.

$\mathrm{H}_{\mathrm{A}}=\mathrm{Si}$ influyen las llantas a manera de recipientes, como factor de riesgo para hospedar al vector.

\section{RESULTADOS}

\section{GRAFICA No 1}

\section{GEOREFERENCIACION DEL MUNICIPIO DE COLCAPIRHUA DEL VALLE BAJO DE LA CIUDAD DE COCHABAMBA}
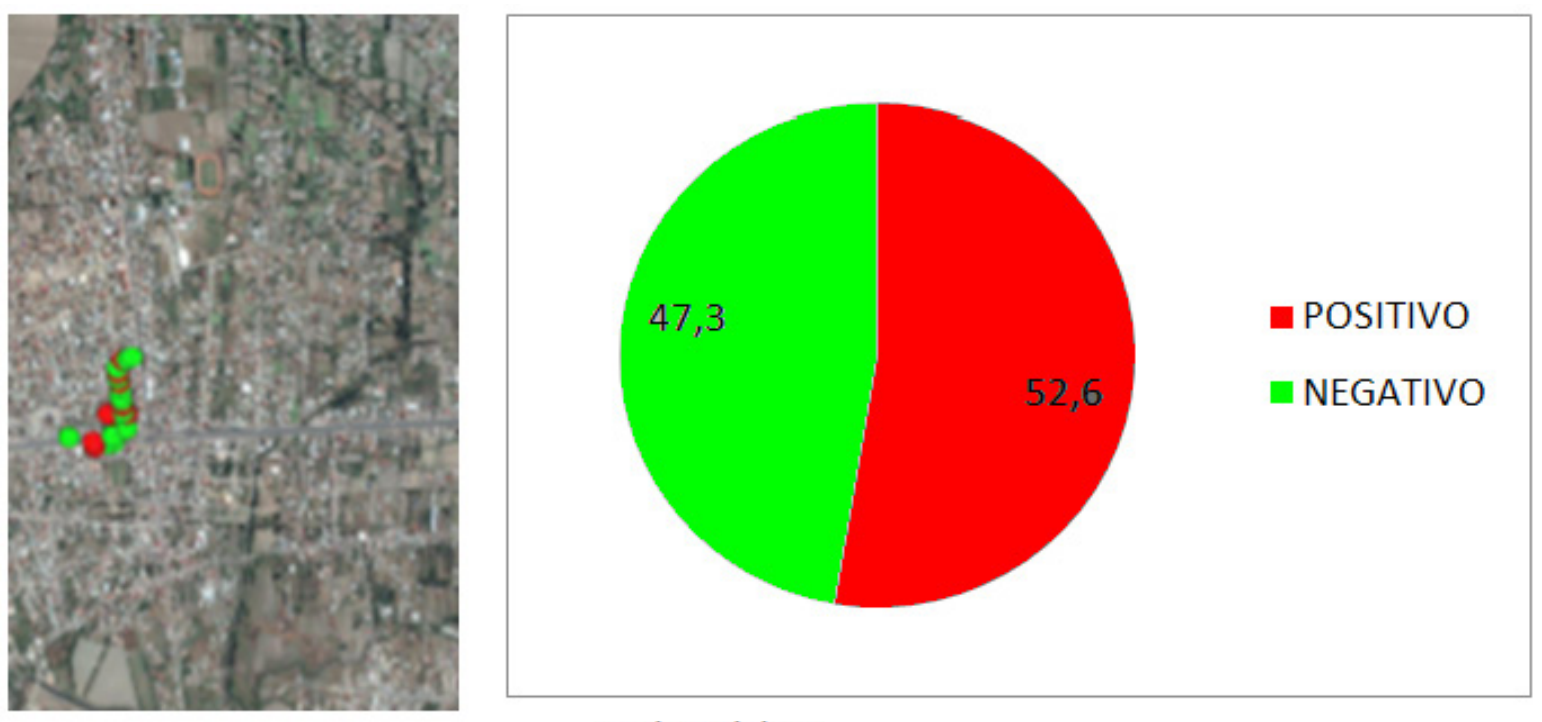

Colcapirhua

Se levantaron datos entomológicos desde San Jose de Kami, pasando por el cementerio de Colcapirhua hacia la avenida principal y la carretera Blanco Galindo que es la carretera troncal interdepartamental, terminando en la Capitán Ustariz; este municipio se encuentra a una altura mínima de $2538 \mathrm{~m} / \mathrm{s} / \mathrm{n} / \mathrm{m}$ siendo esta la altura mas inferior en toda la geo referenciación y a una altura máxima de $2600 \mathrm{~m} / \mathrm{s} / \mathrm{n} / \mathrm{m}$. de todas las casas inspeccionadas en este sector, el $52,6 \%$ se detectaron la presencia de larvas del mosquito $A$. aegypti.

\section{CUADRO $\mathrm{N}^{\circ} 2$}

ÍNDICE DE INFESTACIÓN, RECIPIENTE Y BRETEAU LARVARIA EN VIVIENDAS INSPECCIONADAS

\begin{tabular}{lccc}
\hline & $\begin{array}{c}\text { Índice de } \\
\text { infestación } \\
\text { de larvas }\end{array}$ & $\begin{array}{c}\text { Índice de } \\
\text { infestación de } \\
\text { recipientes }\end{array}$ & $\begin{array}{c}\text { Índice de } \\
\text { infestación de } \\
\text { breteau }\end{array}$ \\
\hline Colcapirhua & $53.3 \%$ & $21.5 \%$ & 1.3 \\
\hline
\end{tabular}


De acuerdo al cuadro el índice de infestación larvaria y de recipientes en los sectores evaluados nos indica un nivel de alarma en toda la región del municipio de Colcapirhua de Cochabamba.

El índice de recipientes en todas las aéreas estudiadas corrobora los índices de infestación y breteau detectados.

Según el índice de breteau, el municipio tiene aproximadamente 1 a más recipientes positivos por casa.

\section{GRAFICA No 2}

\section{ENCUESTA ENTOMOLÓGICA DE LA ZONA DE COLCAPIRHUA}

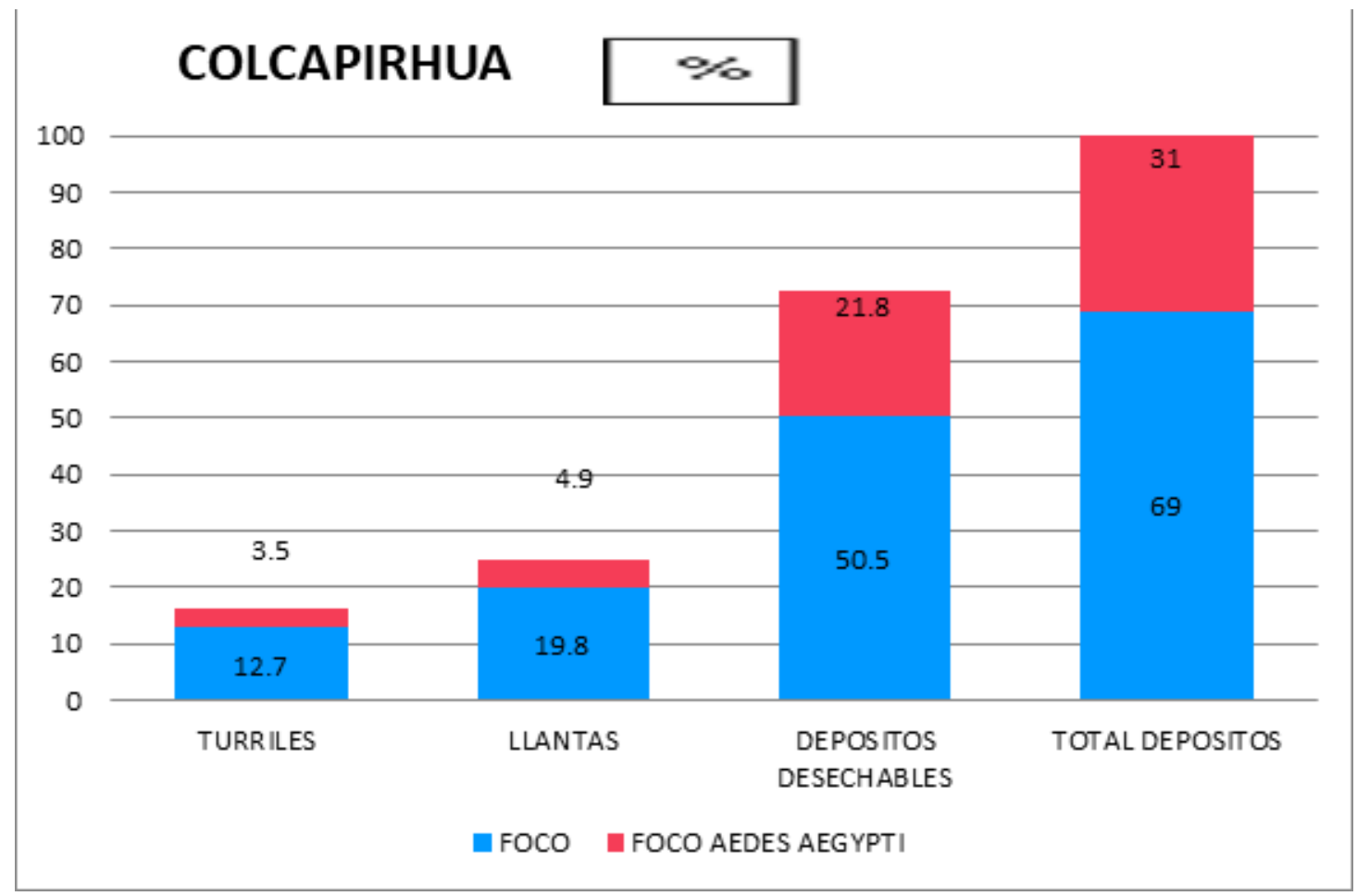

Colcapirhua presenta problemas con presencia de depósitos desechables que sirven de criaderos para el vector, de todos los depósitos estudiados se detectó focos de A. aegypti. Con mayor predominancia en depósitos desechables seguido de llantas, turriles y. como indica el grafico.

CUADRO N ${ }^{\circ} 3$

RIESGO DE CONTACTO ANTE LA PRESENCIA DEL A. aegypti EN EL MUNICIPIO DE COLCAPIRHUA (análisis mediante el riesgo relativo, intervalos de confianza y el test del chi cuadrado con el valor de p.)

\begin{tabular}{|c|c|c|c|c|c|c|c|}
\hline Verificado & $\begin{array}{l}\text { Número } \\
\text { de casas }\end{array}$ & $\begin{array}{c}\%+A \\
\text { aegypti }\end{array}$ & $R R$ & $\begin{array}{c}\text { IC } \\
95 \% \\
\end{array}$ & $x^{2}$ & $P$ & $\begin{array}{l}H_{O=} H_{O=} H_{A} \\
H_{A}=H_{O=} H_{A}\end{array}$ \\
\hline Colcapirhua & 100 & $52,6 \%$ & 3,1 & $\begin{array}{c}1,8- \\
5,3\end{array}$ & 13,5 & & $\mathrm{H}_{\mathrm{A}}$ \\
\hline
\end{tabular}

Según los datos realizados en EPI INFO.

1: RR DE COLCAPIRHUA 
Las personas que viven en Colcapirhua tienen 3 veces más riesgo de albergar el $A$. aegypti en sus casas (RR 3.1) y es estadísticamente significativo (IC: .1,8 - 5,3)

$\mathrm{X}^{2}: \quad \mathrm{H}_{\mathrm{o}}$ No influye la localización de la vivienda en el municipio como factor de riego para albergar al mosquito A.aegypti

$\mathrm{H}_{\mathrm{A}}=\mathrm{SI}$ influye la localización de la vivienda en el municipio como factor de riego para albergar al mosquito A.aegypti

Rechazo mi hipótesis nula $\left(X^{2} 13,5\right)$ y acepto mi hipótesis alternativa; es decir el lugar donde viven si influye como factor de riego para albergar al mosquito A.aegypti en la zona de Colcapirhua. La diferencia observada no es producto del azar y es estadísticamente significativo (p: 0,00009)

\section{CUADRO No 4}

PREDOMINANCIA DE PRESENCIA DE FOCOS LARVARIOS DE AEDES AEGIYTI EN DIFERENTES DEPÓSITOS DE AGUA EN LA REGIÓN DE COLCAPIRHUA (ANÁLISIS MEDIANTE EL RIESGO RELATIVO, INTERVALOS DE CONFIANZA Y EL TEST DEL CHI CUADRADO CON EL VALOR DE P.)

\begin{tabular}{clccccccc}
\hline Verificado & $\begin{array}{l}\text { Depósitos } \\
\text { más frecuen- } \\
\text { tes por zona }\end{array}$ & $N^{\circ}$ & $\%+$ & $R R$ & $I C$ & $X^{2}$ & $P$ & $H_{O=} H_{O=} H_{A}$ \\
\hline $\begin{array}{c}\text { Colcapirhua } \\
\text { total, casa 100 }\end{array}$ & $\begin{array}{l}\text { depósitos } \\
\text { desechables } \\
\text { descartables }\end{array}$ & 41 & $24.3 \%$ & 1,1 & $0,7-1,7$ & 0,2 & 0,331 & $H_{A}=H_{O}=H_{A}$ \\
\hline $\begin{array}{c}\text { Colcapirhua } \\
\text { total, casa 100 }\end{array}$ & Llantas & 35 & $20 \%$ & 0,86 & $0,4-1,6$ & 0,2 & 0,331 & $H_{O}$ \\
\hline
\end{tabular}

\section{2: RR COLCAPIRHUA}

La presencia de depósitos pequeños desechables, en las casas de Colcapirhua tienen 1.1 veces más riesgo de ser hospederos para el mosquito A. aegypti (RR: 1.1). y no es estadísticamente significativo (IC: $0.7-1.7$ ).

$X^{2:} H_{o}=$ No influyen los depósitos pequeños desechables a manera de recipientes, como factor de riesgo para hospedar al mosquito A.aegypti

$\mathrm{H}_{\mathrm{A}}=\mathrm{Si}$ influyen los depósitos pequeños desechables a manera de recipientes, como factor de riesgo para hospedar al mosquito A.aegypti

Rechazo mi hipótesis alternativa y acepto mi hipótesis nula $\left(X^{2:} 0.2\right)$; es decir que no influyen los depósitos pequeños desechables a manera de recipientes como factor de riesgo para hospedar al vector A.aegypti en el municipio de Colcapirhua. La diferencia observada no es producto del azar y no es estadísticamente significativa (p: 0.331).

\section{ANÁLISIS Y DISCUSIÓN}

La rápida proliferación del vector en áreas de clima cálido y baja altitud sobre el nivel del mar, como el oriente boliviano, género la necesidad de analizar la adaptabilidad del A. aegypti en zonas templadas, tal es el caso de la ciudad de Cochabamba, en el municipio de Colcapirhua considerando que este vector transmite diversas enfermedades de morbimortalidad como ser: Dengue, Chikungunya y Zika y otros. 
En febrero de 2016 el Servicio Departamental de Salud (SEDES) Cochabamba identifica por primera vez, seis sectores de la ciudad con colonias de A. aegypti, las mismas que se encuentran distribuidos en los cuatro puntos cardinales de la ciudad como en algunos municipios a la ciudad de Cochabamba ${ }^{7}$. Los hallazgos del presente estudio desarrollado en Colcapirhua confirman la dispersión del A. aegypti mediante la geo referenciación de los focos identificados del vector, con algunos sectores con mayor prevalencia. De la misma forma, se evidencio que la dispersión del vector en el área de estudio alcanzo una altura mínima de $2538 \mathrm{~m} / \mathrm{s} / \mathrm{n} / \mathrm{m}$. y una altura máxima de 2623 $\mathrm{m} / \mathrm{s} / \mathrm{n} / \mathrm{m}$ para la colonización de larvas de A. aegypti.

De 100 Casas inspeccionados en el municipio de Colcapirhua 52 casas son positivas al A.egypti, que corresponde al $52 \%$. De acuerdo a los índices epidemiológicos de la OMS Cochabamba ha estado cursando un nivel de alarma con una dispersión generalizada en el cercado urbano y el valle bajo urbano, no en tanto el IB que nos indica productividad de larvas en viviendas positivas se mantuvo en un nivel óptimo lo que representa probablemente una densidad poblacional relativamente baja de larvas de A. aegypti.

De acuerdo al análisis estadístico referente a la infestación larvaria de A. aegypti, se identificó que hay 3 veces más riesgo de vivir en Colcapirhua.

En la provincia de Limón, en Costa Rica, se evidenció que los sitios de cría más utilizados por A. aegypti fueron llantas y tanques. De la misma forma, en la Ciudad de La Habana, Cuba, localizaron que los depósitos más productores de A. aegypti fueron los tanques bajos, llantas y bebederos; mientras que, en Mérida, Venezuela, fueron las Ilantas, tanques, macetas, floreros. En Yucatán, México, se reportaron las cubetas, que representan (38 \%) de los criaderos ${ }^{53,56,47}$. en el municipio de lisa perteneciente a la Habana Cuba la mayor positividad fueron los de almacenamiento de agua y artificiales no útiles los recipientes asociados a la mayor infestación por $\mathrm{A}$. aegypti. ${ }^{54,55}$. Todos datos resultados corroboran los hallazgos encontrados en el área de estudio, los recipientes que encontramos con mayor frecuencia al vector son en turriles, llantas y depósitos pequeños desechables.

Según otra investigación la provincia de Limón del país de Costa Rica Los sitios de cría más frecuentes fueron las llantas, según el estudio se encuentran año tras año los mismos sitios de cría del A. aegypti tanto en cantidad como en calidad y esta proliferación la relacionan directamente por los malas prácticas y malos hábitos, y por la baja calidad de los servicios de salud ${ }^{57}$. Según nuestro estudio, las llantas son un factor de riesgo para la propagación y adaptación del vector (Llantas: $\mathrm{RR} 0,86, \mathrm{IC} .0 .4-1.6, \mathrm{X}^{2}$ $=0.2, P=0.331$ )

La presencia de llantas en las casas, tienen una probabilidad de 1 veces más riesgo de hospedar al A. aegypti en los hogares y la localidad. Si influyen las llantas a manera de recipientes, como factor de riesgo para hospedar al mosquito $A$. aegypti

\section{CONCLUSIONES}

El vector puede adaptarse a climas templados hasta los $2600 \mathrm{~m} / \mathrm{s} / \mathrm{n} / \mathrm{m}$. y está distribuido en forma general en todo el municipio de Colcapirhua

De acuerdo a los índices epidemiológicos de la OMS el municipio de Colcapirhua en el periodo de estudio ha estado cursando un nivel de alarma con una dispersión ge- 
neralizada en el municipio, no en tanto el IB que nos indica productividad de larvas en viviendas positivas se mantuvo en un nivel óptimo lo que representa probablemente una densidad poblacional relativamente baja de larvas de A. aegypti.

Colcapirhua al ser parte del eje troncal entre La Paz y Santa Cruz es un punto crítico para la dispersión de las enfermedades causadas por el A. egypti, considerando que el vector está presente en cercanías a las principales carreteras que unen oriente y occidente.

Los depósitos más importantes que probablemente influenciaron en la adaptación del A. aegypti fueron llantas, turriles de almacenamiento de agua que muy frecuentemente son transportados de un lugar a otro del país.

\section{BIBLIOGRAFÍA}

1. OMS | El mosquito [Internet]. WHO. [cited 2017 Sep 12]. Available from: http:// www.who.int/denguecontrol/mosquito/es/

2. Mora-Covarrubias A de la, - FJ-V, - SM-A. Distribución geoespacial y detección del virus del dengue en mosquitos Aedes (Stegomyia) aegypti de Ciudad Juárez, Chihuahua, México. Salud Pública México. 2010;52(2):127-33.

3. Biologia do Aedes [Internet]. Aedes aegypti - Introdução aos Aspectos Científicos do Vetor. [cited 2017 Mar 12]. Available from: http://auladengue.ioc.fiocruz. $\mathrm{br} / \mathrm{p}=72$

4. OPS/OMS. Enfermedades infecciosas Dengue Guía para el equipo de salud [Internet]. 3rd ed. Vol. 2. Cdad. Autónoma de Bs. As., República Argentina: Ministerio de salud Presidencia de la nación; 2013. Available from: http://www.msal.gob. ar/images/stories/epidemiologia/pdf/guia-dengue.pdf

5. Marquetti M del C, Suárez S, Bisset J, Leyva M. Reporte de hábitats utilizados por Aedes aegypti en Ciudad de La Habana, Cuba. Rev Cuba Med Trop [Internet]. 2005 Aug [cited 2017 Dec 11];57(2). Available from: http://scielo.sld.cu/scielo.php?script=sci_arttext\&pid=S0375-07602005000200013\&lng=es\&nrm=iso\&tlng=es

6. Vallejo Castro E, Quiñones López A. BROTE EPIDÉMICO DE DENGUE EN LA LOCALIDAD DE OMEREQUE DEPARTAMENTO DE COCHABAMBA FEBRERO 2009. Rev Médica Cochabamba. /;64.

7. Castro T. Insecto transmisor del zika está en 6 sitios de las 4zonas se la ciudad. Opinion. Edicion Impresa. 2016 Feb 16;1.

8. Cadavid R, Fernando J. Aspectos entomológicos del Dengue. Infectio [Internet]. 2011 Oct 27 [cited 2016 Mar 12];8(3). Available from: http://www.revistainfectio. org/site/portals/0/ojs/index.php/infectio/article/view/252

9. Manual vigilancia Aa final13 de junio - Manual de Vigilancia y Control de Aedes aegypti 2011_0.pdf [Internet]. [cited 2017 Jul 13]. Available from: http://www.msp. gub.uy/sites/default/files/archivos_adjuntos/Manual\%20de $\% 20$ Vigilancia $\% 20$ y\%20Control\%20de\%20Aedes\%20aegypti\%202011_0.pdf

10. CARACTERÍSRICAS DEL MOSQUITO [Internet]. [cited 2017 Aug 29]. Available from: http://www.ceibal.edu.uy/contenidos/areas_conocimiento/cs_naturales/ dengue_090225/mosqui.htm

11. HÁBITAT [Internet]. [cited 2016 Aug 29]. Available from: http://www.ceibal.edu.uy/

Fuentes predominantes de la colonización de Aedes aegypti que facilita el proceso de dispersión en el municipio de Colcapirhua 
contenidos/areas_conocimiento/cs_naturales/dengue_090225/habitat.htm

12. Santacoloma Varón L, Chaves Córdoba B, Brochero HL. Susceptibility of Aedes aegypti to DDT, deltamethrin, and lambda-cyhalothrin in Colombia. Rev Panam Salud Pública. 2010 Jan;27(1):66-73.

13. 79-Aedes_aegypti.pdf [Internet]. [cited 2017 Jul 13]. Available from: http://www. produccion-animal.com.ar/fauna/Fauna_insectos/79-Aedes_aegypti.pdf

14. Aedes aegypti - EcuRed [Internet]. [cited 2017 Aug 29]. Available from: http:// www.ecured.cu/Aedes_aegypti

15. Layout 1 - index.php [Internet]. [cited 2017 Jul 13]. Available from: http://www. paho.org/hq/index.php?option=com_docman\&task=doc_view\&Itemid=270\&gi$\mathrm{d}=27234$ \&lang $=e$ s

16. libro entomologia contenido.cdr - Anexo 14I2 COL M\&E 2009.pdf [Internet]. [cited 2017 Jul 13]. Available from: http://www.orasconhu.org/documentos/Anexo\%20 14I2\%20\%20COL\%20M\&E\%202009.pdf

17. Guía Metodológica para Vigilancia Entomológica con Ovitrampas.docx - guia_vigilancia_entomologica_ovitrampas.pdf [Internet]. [cited 2016 Jul 13]. Available from: http://www.gob.mx/cms/uploads/attachment/file/37865/guia_vigilancia_ entomologica_ovitrampas.pdf

18. Quispe E, Carbajal A, Gozzer J, Moreno B. Ciclo biológico y Tabla de Vida de Aedes aegypti, en laboratorio: Trujillo (Perú), 2014. Rev REBIOLEST. 2015 Jul 20;3(1):91-101.

19. Marquetti M del C, Valdés $V$, Aguilera L, Navarro A. Vigilancia entomológica de Aedes (S) aegypti y otros culícidos en Ciudad de La Habana, Cuba 1991-1996. Rev Cubana Med Trop. 2000 Aug;52(2):133-7.

20. Forattini $\mathrm{OP}$, Brito $M$ de. Reservatórios domiciliares de água e controle do Aedes aegypti. Rev Saúde Pública. 2003 Oct 1;37(5):676-7.

21. Bolivia: Zika mosquito adapting to life at 2,600 meters above sea level [Internet]. H5N1. [cited 2017 Sep 12]. Available from: http://crofsblogs.typepad.com/ h5n1/2016/03/bolivia-zika-mosquito-adapting-to-life-at-2600-meters-above-sealevel.html

22. Rodríguez Cruz R. Estrategias para el control del dengue y del Aedes aegypti en las Américas. Rev Cubana Med Trop. 2002 Dec;54(3):189-201.

23. Pública BM de PS y S. Vigilancia y control del aedes aegypti en Bolivia. Bol Epidemiol Wash. 1982;3(2):7.

24. Mitchell C. Bolivia [Internet]. 2013 [cited 2017 oct 12]. Available from: file:///C:/ Users/ASUS/Desktop/Bolivia.htm

25. Guidebolivia: Enfermedades con riesgo en Bolivia [Internet]. [cited 2017 Nov 12]. Available from: http://www.guidebolivia.com/pratique/es_maladie.htm

26. Omereque. OMEREQUE UN LUGAR POR DESCUBRIR: dengue infecta a 60 personas en omereque [Internet]. OMEREQUE UN LUGAR POR DESCUBRIR. 2009 [cited 2017 Mar 5]. Available from: http://omereque.blogspot.com/2009/02/ dengue-infecta-60-personas-en-omereque.html

27. Brady OJ, Gething PW, Bhatt S, Messina JP, Brownstein JS, Hoen AG, et al. Refining the Global Spatial Limits of Dengue Virus Transmission by Evidence-Based 
Consensus. Reithinger R, editor. PLoS Negl Trop Dis. 2012 Aug 7;6(8):e1760.

28. Plan de acción para fortalecer la vigilancia, control y prevención del dengue, será socializado en Cochabamba [Internet]. ReliefWeb. 2012 [cited 2017 Dec 17]. Available from: http://reliefweb.int/report/bolivia/plan-de-acci\%C3\%B3n-para-fortalecer-la-vigilancia-control-y-prevenci\%C3\%B3n-del-dengue-ser\%C3\%A1

29. OMS | Chikungunya [Internet]. WHO. [cited 2017 Dec 14]. Available from: http:// www.who.int/mediacentre/factsheets/fs327/es/

30. Zika, el virus que provoca graves malformaciones en bebés, se extiende por América [Internet]. abc. [cited 2017 Dec 14]. Available from: http://www.abc.es/ sociedad/abci-zika-virus-provoca-graves-malformaciones-bebes-extiende-america-201512080237_noticia.html

31. Alerta Epidemiológica, Infección por virus Zika [Internet]. 2015 [cited 2017 Dec 14]. Available from: http://www.paho.org/par/index.php?option=com_content\&view=article\&id=1319:alerta-epidemiologica-infeccion-por-virus-zika\&ltemid=258

32. Caprara A, Lima JWDO, Peixoto ACR, Motta CMV, Nobre JMS, Sommerfeld J, et al. Entomological impact and social participation in dengue control: a cluster randomized trial in Fortaleza, Brazil. Trans R Soc Trop Med Hyg. 2015 Feb 1;109(2):99-105.

33. Quintanilla SD, Barruetos E. Fiebre Chikungunya. Acta Pediátrica Hondureña. 2015 Dec 8;5(1-2):371-7.

34. Montero A. Fiebre chikungunya - Una nueva amenaza global. Med Clínica. 2015 Agosto;145(3):118-23.

35. Conde M, Orjuela LI, Castellanos CA, Herrera-Varela M, Licastro S, Quiñones ML. Evaluación de la sensibilidad a insecticidas en poblaciones de Aedes aegypti (Diptera: Culicidae) del departamento de Caldas, Colombia, en 2007 y 2011. Biomédica. 2015 Mar;35(1):43-52.

36. Oster AM, Brooks JT, Stryker JE, Kachur RE, Mead P, Pesik NT, et al. Interim Guidelines for Prevention of Sexual Transmission of Zika Virus - United States, 2016. MMWR Morb Mortal Wkly Rep. 2016 Feb 12;65(5):120-1.

37. Carvajal AC, Peña SO, L JFO. Infección por Virus Zika (VZIK): Arbovirosis emergente en las Américas. Med Interna [Internet]. 2015 Jan 7 [cited 2017 Aug 30];31(1). Available from: http://www.svmi.web.ve/ojs/index.php/medint/article/ view/40

38. Hennessey M, Fischer M, Staples JE. Zika Virus Spreads to New Areas - Region of the Americas, May 2015-January 2016. Am J Transplant. 2016 Mar 1;16(3):1031-4.

39. Dirlikov E, Ryff KR, Torres-Aponte J, Thomas DL, Perez-Padilla J, Munoz-Jordan J, et al. Update: Ongoing Zika Virus Transmission - Puerto Rico, November 1, 2015-April 14, 2016. MMWR Morb Mortal Wkly Rep. 2016 May 6;65(17):451-5.

40. Reyes ER, Rodríguez NMO. Teratogénesis por acción viral. Rev Electrónica Dr Zoilo E Mar Vidaurreta [Internet]. 2016 Mar 15 [cited 2017 Aug 30];41(5). Available from: http://revzoilomarinello.sld.cu/index.php/zmv/article/view/702

41. Petersen EE, Staples JE, Meaney-Delman, D, Fischer M, Ellington SR, Callaghan WM, et al. Interim Guidelines for Pregnant Women During a Zika Virus Outbreak — United States, 2016. MMWR Morb Mortal Wkly Rep. 2016 Jan 22;65(2):30-3.

Fuentes predominantes de la colonización de Aedes aegypti que facilita el proceso de dispersión en el municipio de Colcapirhua 
42. Sanchez JD. OPS OMS | Educación en inocuidad de alimentos: Investigación de Conocimientos Actitudes y Prácticas (CAP) [Internet]. Pan American Health Organization / World Health Organization. 2016 [cited 2017 Aug 30]. Available from: http:// www.paho.org/hq/index.php?option=com_content\&view=article\&id=10493\%3Aeducacion-inocuidad-alimentos-investigacion-conocimientos-actitudes-practicas-cap\&catid=1237\%3Aeducation-on-food-safety\&ltemid=41279\&lang=es

43. Aldaz $\mathrm{S}$, Katerine $\mathrm{C}$. Conocimientos prácticos y aptitudes en relación a medidas para el dengue en la población del barrio Rayito de Luz del cantón Machala en el primer semestre de laño 2013. 2014 [cited 2017 Aug 29]; Available from: http:// repositorio.utmachala.edu.ec/handle/48000/3863

44. Castro Peraza M, Gálvez Miranda C, Sanchez Váldes L, Pérez Chacón D, Polo Díaz V, Concepción Díaz D, et al. Encuesta poblacional sobre conocimientos y percepciones acerca de dengue contra prácticas preventivas en el municipio Lisa. Rev Cubana Med Trop. 2010 Dec;62(3):245-53.

45. Paico-Romero C, Polo-Capuñay A, Díaz-Exebio G, Díaz-Vélez C. Validación y desarrollo del instrumento: Nivel de conocimientos en diagnóstico y tratamiento sobre dengue en médicos de la región Lambayeque. Acta Médica Peru. 2012 Jul;29(3):143-7.

46. González Morales A, Sala I, María A. Nivel de conocimientos, actitudes y prácticas sobre la prevención del mosquito Aedes aegypti en comunidades del municipio Diez de Octubre, La Habana. Rev Cuba Hig Epidemiol. 2011 Aug;49(2):247-59.

47. Diéguez Fernández L, Fernández C, María S, Prada Noy Y, Cruz Pineda C, Rodríguez de la Vega R. Aedes (St.) aegypti en tanques bajos y sus implicaciones para el control del dengue en Camagüey. Rev Cubana Med Trop. 2010 Aug;62(2):937.

48. Chuc S, Hurtado-Díaz M, Schilmann A, Riojas-Rodríguez H, Rangel H, González-Fernández MI. Local conditions of vulnerability associated with dengue in two communities of Morelos. Salud Pública México. 2013 Apr;55(2):170-8.

49. Espinoza Gómez F, Suárez H, Moisés C, Coll Cárdenas R. Factors that modify the larval indices of Aedes aegypti in Colima, Mexico. Rev Panam Salud Pública. 2001 Jul;10(1):6-12.

50. Benítez-Leite S, Machi ML, Gibert E, Rivarola K. Conocimientos, actitudes y prácticas acerca del dengue en un barrio de Asunción. Rev Chil Pediatría. 2002 Jan;73(1):64-72.

51. COCHABAMBA POR MUNICIPIOS - CNPV 2012 | CENSOS [Internet]. [cited 2017 Sep 12]. Available from: http://censosbolivia.ine.gob.bo/content/cochabamba-por-municipios-cnpv-2012

52. Chang Camero Y, Lugo Mendosa J, Barceló Rodríguez G, Martínez García J, Sautie C, Isabel M. Depósitos observados con larvas de Aedes aegypti durante el uso operacional del biolarvicida Bactivec. Rev Cubana Med Trop. 2013 Apr;65(1):90-8.

53. Carrazana Trujillo $M$, Fernández $M$, Carmen $M$ del, Vázquez Cáceres $A$, Montano $\mathrm{M}$ de $\mathrm{O}$, Luis J. Dinámica estacional y temporal de Aedes aegypti (Diptera:Culicidae) en el municipio Cienfuegos. Rev Cubana Med Trop. 2010 Aug;62(2):98-106.

54. Fernández M, Carmen M del, Leyva Silva M, Bisset Lazcano J, García Sol A. Re- 
cipientes asociados a la infestacion por Aedes aegypti en el municipio Lisa. Rev Cubana Med Trop. 2009 Dec;61(3):232-8.

55. J PE. Factores asociados a la infestación intradomiciliaria por Aedes aegypti en el distrito de Tambogrande, Piura 2004. Rev Peru Med Exp Salud Publica [Internet]. 2016 Sep 5 [cited 2017 Sep 13];24(2). Available from: http://www.perurevista.com/index.php/publica/article/view/5575

56. Bisset Lazcano JA, Marquetti MDC, Portillo R, Rodríguez MM, Suárez S, Leyva M. Factores ecológicos asociados con la presencia de larvas de Aedes aegypti en zonas de alta infestación del municipio Playa, Ciudad de La Habana, Cuba. Ecological factors linked to the presence of Aedes aegypti larvae in highly infested areas of Playa, a municipality belonging to Ciudad de La Habana, Cuba [Internet]. 2006 [cited 2017 Sep 13]; Available from: http://iris.paho.org/xmlui/handle/123456789/7962

57. Marín Rodríguez R, Díaz Ríos M, Álvarez Gutiérrez Y, Calderón Arguedas O. Sitios de cría de Aedes aegypti (Linnaeus) y distribución geográfica de Aedes albopictus (Skuse) en la provincia de Limón, Costa Rica. 2012. Rev Cubana Med Trop. 2014 Aug;66(2):219-27.

58. Marquetti M del C, Bisset J, Portillo R, Rodríguez M, Leyva M. Factores de riesgo de infestación pupal con Aedes aegypti dependientes de la comunidad en un municipio de Ciudad de La Habana. Rev Cubana Med Trop. 2007 Apr;59(1):0-0. 